OPEN ACCESS

Edited by:

Qinglin Meng,

South China University of

Technology, China

Reviewed by:

Wei Qiu,

State University of New York,

United States

Fei Ji,

PLA General Hospital, China

*Correspondence:

Zhiwu Huang

huangzw86@126.com

Hao Wu

wuhao@shsmu.edu.cn

tThese authors have contributed equally to this work and share first authorship

Specialty section

This article was submitted to Family Medicine and Primary Care,

a section of the journal

Frontiers in Medicine

Received: 31 January 2021 Accepted: 02 March 2021

Published: 25 March 2021

Citation:

Wang Q, Qian M, Yang L, Shi J, Hong Y, Han K, Li C, Lin J, Huang Z and Wu H (2021) Audiometric

Phenotypes of Noise-Induced Hearing Loss by Data-Driven Cluster Analysis and Their Relevant Characteristics.

Front. Med. 8:662045.

doi: 10.3389/fmed.2021.662045

\section{Audiometric Phenotypes of Noise-Induced Hearing Loss by Data-Driven Cluster Analysis and Their Relevant Characteristics}

\author{
Qixuan Wang ${ }^{1,2,3+}$, Minfei Qian ${ }^{1,2,3 \dagger}$, Lu Yang ${ }^{1,4}$, Junbo Shi ${ }^{1,4}$, Yingying Hong ${ }^{1,4}$, \\ Kun Han ${ }^{1,2,3}$, Chen $\mathrm{Li}^{5}$, James Lin ${ }^{5}$, Zhiwu Huang ${ }^{1,2,3,4^{*}}$ and Hao Wu ${ }^{1,2,3^{*}}$
}

${ }^{1}$ Department of Otolaryngology-Head and Neck Surgery, Ninth People's Hospital, Shanghai Jiao Tong University School of Medicine, Shanghai, China, ${ }^{2}$ Ear Institute, Shanghai Jiao Tong University School of Medicine, Shanghai, China, ${ }^{3}$ Shanghai Key Laboratory of Translational Medicine on Ear and Nose Diseases, Shanghai, China, ${ }^{4}$ Hearing and Speech Center, Ninth People's Hospital, Shanghai Jiao Tong University School of Medicine, Shanghai, China, ${ }^{5}$ Network and Information Center, Shanghai Jiao Tong University, Shanghai, China

Background: The definition of notched audiogram for noise-induced hearing loss (NIHL) is presently based on clinical experience, but audiometric phenotypes of $\mathrm{NIHL}$ are highly heterogeneous. The data-driven clustering of subtypes could provide refined characteristics of $\mathrm{NIHL}$, and help identify individuals with typical NIHL at diagnosis.

Methods: This cross-sectional study initially recruited 12,218 occupational noise-exposed employees aged 18-60 years from two factories of a shipyard in Eastern China. Of these, 10,307 subjects with no history of otological injurie or disease, family history of hearing loss, or history of ototoxic drug use were eventually enrolled. All these subjects completed health behavior questionnaires, cumulative noise exposure (CNE) measurement, and pure-tone audiometry. We did data-driven cluster analysis (k-means clustering) in subjects with hearing loss audiograms $(n=6,599)$ consist of two independent datasets $(n=4,461$ and $n=2,138)$. Multinomial logistic regression was performed to analyze the relevant characteristics of subjects with different audiometric phenotypes compared to those subjects with normal hearing audiograms ( $n=3,708)$.

Results: A total of 10,307 subjects (9,165 males [88.9\%], mean age 34.5 [8.8] years, mean CNE 91.2 [22.7] dB[A]) were included, 3,708 (36.0\%) of them had completely normal hearing, the other 6,599 (64.0\%) with hearing loss audiograms were clustered into four audiometric phenotypes, which were replicable in two distinct datasets. We named the four clusters as the 4-6 kHz sharp-notched, 4-6 kHz flat-notched, 3-8 kHz notched, and $1-8 \mathrm{kHz}$ notched audiogram. Among them, except for the $4-6 \mathrm{kHz}$ flat-notched audiogram which was not significantly related to $\mathrm{NIHL}$, the other three phenotypes with different relevant characteristics were strongly associated with noise exposure. In particular, the $4-6 \mathrm{kHz}$ sharp-notched audiogram might be a typical subtype of $\mathrm{NIHL}$. 


\begin{abstract}
Conclusions: By data-driven cluster analysis of the large-scale noise-exposed population, we identified three audiometric phenotypes associated with distinct $\mathrm{NIHL}$ subtypes. Data-driven sub-stratification of audiograms might eventually contribute to the precise diagnosis and treatment of $\mathrm{NIHL}$.
\end{abstract}

Keywords: noise-induced hearing loss, audiometric phenotype, notched audiogram, unsupervised learning, data-driven cluster analysis, multivariate characteristics

\section{INTRODUCTION}

Noise-induced hearing loss (NIHL) is one of the most common hearing loss in adults (1), with increasing incidence in children and adolescents (2) due to widespread recreational and transport noise exposure $(3,4)$. The World Health Organization (WHO) estimates that $10 \%$ of the world population is exposed to sound levels that could potentially cause NIHL (5). To date, treatment options for NIHL are limited, while $\sim 50 \%$ of this burden could be prevented by early detection of NIHL, avoidance of noise exposure, prompt intervention, etc. (6).

It is widely accepted that the noise exposure usually causes high-frequency sensorineural hearing impairment ( 7 , 8). Despite several previously concluded abstract phenotypes of NIHL including the high-frequency audiometric notch and the bulge downwards audiogram (9), there are still no clear audiometric criteria on stratifications of NIHL, which makes it difficult to specifically evaluate NIHL during clinical and primary health care $(10,11)$. One reason for this is the heterogeneous audiometric phenotypes of NIHL, involving complex confounding influencing factors. The majority of studies have adopted different definitions of high-frequency hearing loss $(12,13)$ and notched audiogram $(14-16)$, which were chosen mainly by specialized intuition or clinical experience, rather than by data-driven analysis. These inconsistent assessment methods were manifested by various ranges of frequency and degrees of hearing loss, which may represent different subtypes of NIHL with inconsistent responses to intervention, and inevitably result in incomparable conclusions between studies.

Generally, descriptions of NIHL phenotypes are limited by subjectivity and poor data support. A data-driven classification that incorporates the multifrequency audiogram of NIHL is needed to identify subtypes with consistent patterns and characteristics. Cluster analysis is an unsupervised exploratory data mining technique able to group the most similar individuals with multiple specified variables in the same group called "cluster" without any previously defined hypothesis (17). Since audiogram stratification is based on the complex non-linear combination of thresholds at several frequencies, unbiased datadriven cluster analysis has recently been found to be a useful method for the identification of audiometric phenotypes (18, 19). We postulated that cluster analysis could be applied for classifying audiograms of NIHL.

In the current study, based on audiograms of 10,307 Chinese shipyard employees with various noise exposure levels, we used the k-means clustering algorithm to classify subtypes of NIHL in two distinct noise-exposed populations from different factories.
The confounding influencing factors related to these subtypes were further analyzed to optimize the assessment for different subtypes of NIHL, which could provide a powerful tool to identify those individuals at great risk of NIHL and guide optimal prevention of noise exposure.

\section{METHODS}

\section{Study Population}

We conducted this hearing and health investigation in a shipyard in eastern China from August 1, 2017, to June 30, 2018. A total of 12,218 subjects aged $18-60$ years were initially recruited, and 10,307 from two steel factories (6,631 from factory 1 , and 3,676 from factory 2) were included in the analysis based on the following criteria: (1) completed questionnaire and audiometric data, (2) no history of otological injuries or diseases, (3) no family history of hearing loss, (4) no history of ototoxic drug use, (5) no profound hearing loss (average threshold at $0.5-2 \mathrm{kHz}$ frequencies $>70 \mathrm{~dB} \mathrm{HL}$ in any ear), and (6) no perforation of tympanic membrane or abnormal tympanogram. Sex and race were self-reported. Figure 1 shows the flowchart of this cross-sectional study, which was in accordance with the Strengthening the Reporting of Observational Studies in Epidemiology (STROBE) reporting guidelines and approved by the ethics committee of the Ninth People's Hospital affiliated to Shanghai Jiao Tong University School of Medicine. All the participants signed written informed consent forms.

\section{Audiometry}

Pure-tone air-conduction audiometry at frequencies of 0.5 , $1,2,3,4,6$, and $8 \mathrm{kHz}$ in both ears was performed by certified audiological technicians using an audiometer (Otometrics Madsen, Xeta, Denmark) with TDH-39P headsets in a soundproof booth in accordance with the regulations of ISO 8253-1: 2010. The subjects were not exposed to occupational noise or loud sounds within $16 \mathrm{~h}$ before being examined. The average threshold of the left and right ears at each frequency was calculated for subsequent analysis without the age-correction according to ISO 7029: 2017, in order to avoid the artificial modification on the subsequent cluster analysis. Normal hearing was defined as hearing threshold $\leq 25 \mathrm{~dB}$ HL over $0.5-8 \mathrm{kHz}$ frequencies. Hearing loss was defined as hearing threshold $>25$ $\mathrm{dB}$ HL at any frequency.

\section{Questionnaire}

Demographic variables (sex, age, race, job type, working time-length) and behavioral characteristics, including hearing 


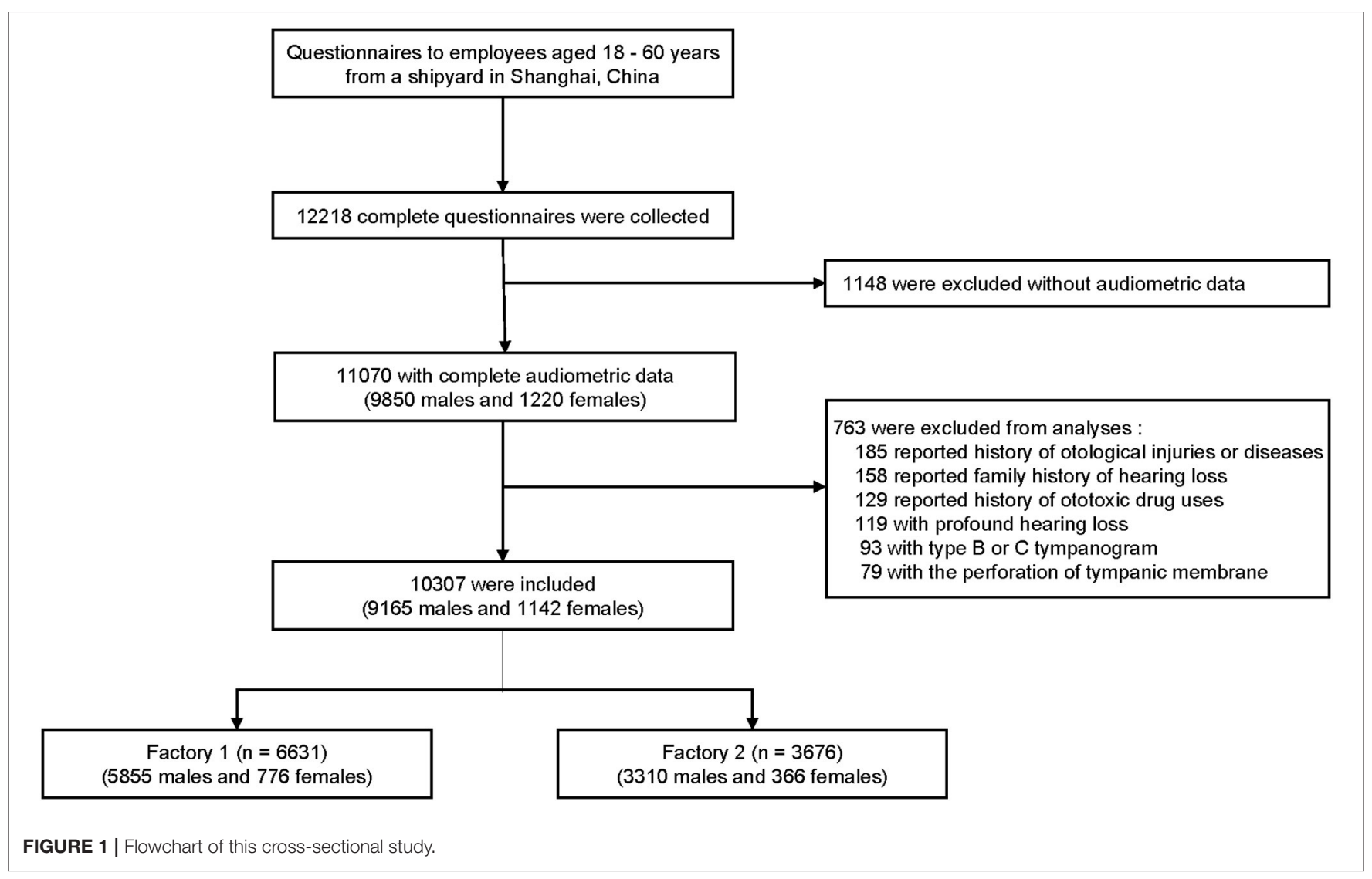

protection device (HPD) use ( $<4 \mathrm{~h} /$ work-day, $\geq 4 \mathrm{~h} /$ work-day), personal earphone use $(<1 \mathrm{~h} /$ day, $\geq 1 \mathrm{~h} /$ day $)$, tobacco $(<10$ cigarettes/day, $\geq 10$ cigarettes/day) and alcohol ( $<50 \mathrm{~g} /$ day, $\geq 50$ g/day) consumption, and auditory-related symptoms (hearing difficulty and tinnitus), were collected through a self-reported questionnaire. Body mass index (BMI) was measured and calculated by investigators, and then categorized into non-obese $\left(<28 \mathrm{~kg} / \mathrm{m}^{2}\right)$ and obese $\left(\geq 28 \mathrm{~kg} / \mathrm{m}^{2}\right)$ groups.

\section{Noise Exposure Dose}

A composite quantitative noise exposure index, the cumulative noise exposure (CNE), was used to estimate the noise exposure level for each subject, which was calculated using the following formula (20):

$$
\mathrm{CNE}=L_{\mathrm{Aeq}, 8 \mathrm{~h}}+10 \log T,
$$

where $L_{\mathrm{Aeq}, 8 \mathrm{~h}}$ is the equivalent sound pressure level in A weight of 8 continuous hours of a work-day, which was measured and analyzed using the personal exposure dosimeter (Aihua, ASV5910 type, Hangzhou, China). Subjects were required to wear the dosimeter on the shoulder for five work-days to calculate the average $L_{\mathrm{Aeq}, 8 \mathrm{~h}} . T$ is the working time-length in years obtained from the questionnaire.

\section{Data-Driven Cluster Analysis}

Seven variables including standardized values of thresholds at frequencies of $0.5,1,2,3,4,6$, and $8 \mathrm{kHz}$ were input for $\mathrm{k}$ means cluster analysis performed using $\mathrm{R}$ software (version 4.0.3) (21). The optimal number of clusters was selected according to the within cluster sum of squares (WSS) (22), the number of clusters from 2 to 15 was tried, and the last one that significantly reduced the WSS (at the inflection point of the curve) was selected as the optimal number of clusters (Figures 2A-E). Datadriven cluster analysis was performed in data from two factories (dataset 1 and dataset 2) separately, and then repeated in the total data.

\section{Statistical Analysis}

Data analysis was performed by using IBM SPSS version 24.0 software (SPSS Inc., Chicago, IL, USA) except for cluster analysis. Continuous variables are expressed as the mean (standard deviation, SD), and categorical variables are presented as percentages $(n[\%])$. Statistical significance for differences in continuous variables was examined using Student's $t$ test (between dataset 1 and dataset 2) or ANOVA (between cluster subtypes with Dunn-Bonferroni tests for posthoc analyses), and categorical variables were compared by the chi-square test. Multinomial logistic regression models were used to analyze relevant factors of different clusters of audiometric subtypes. For the hierarchical regression, age was categorized into 3 groups $(<30,30-45$, and $>45$ years). For 
A

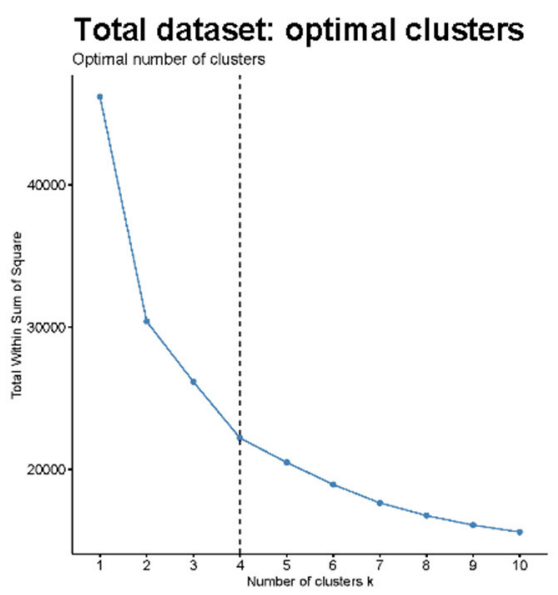

C

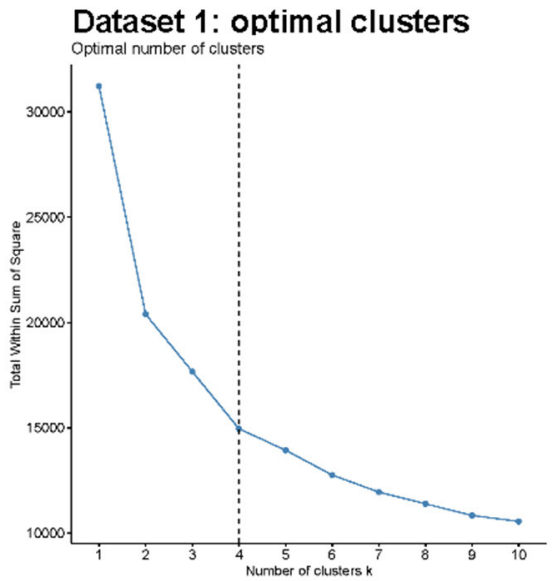

E

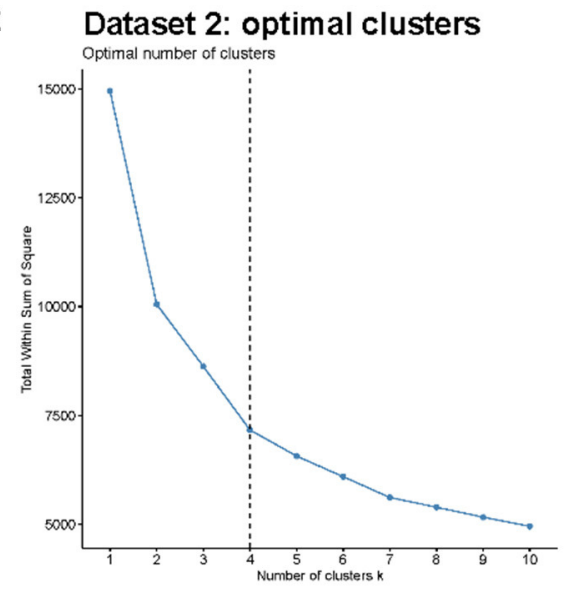

B

Audiometric clusters of total dataset

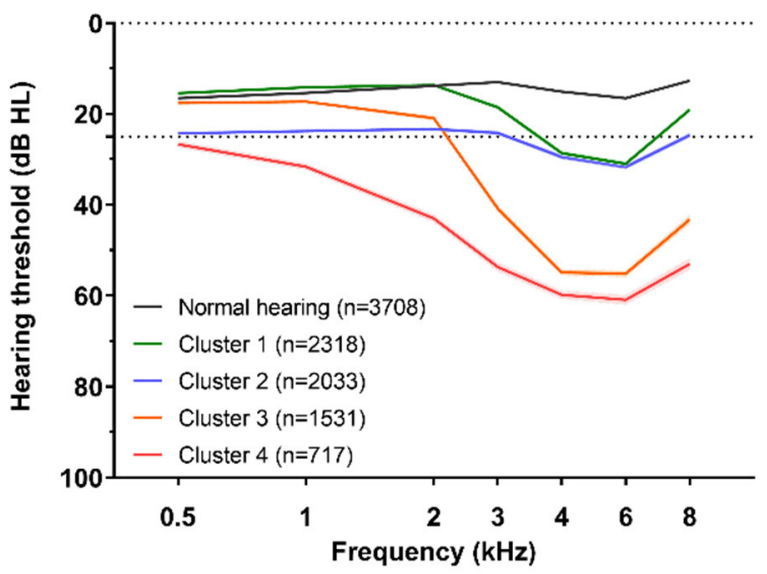

D

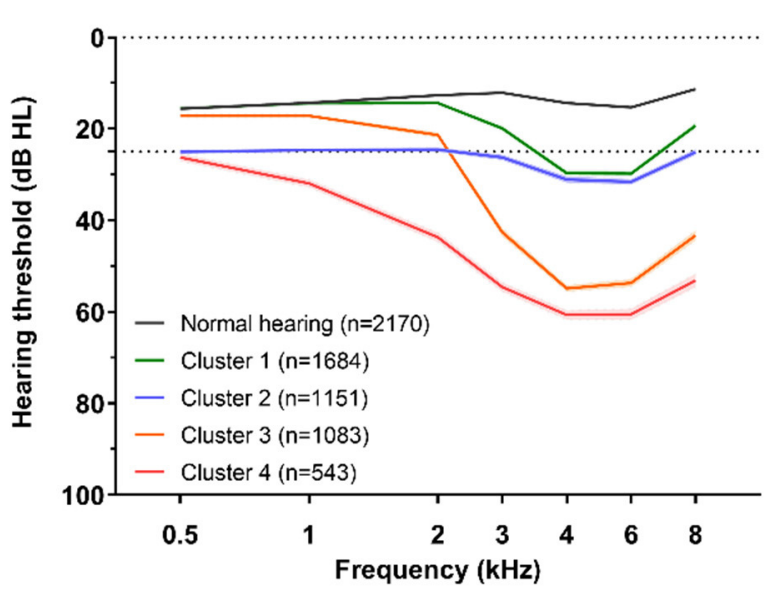

$\mathbf{F}$

Audiometric clusters of dataset 2

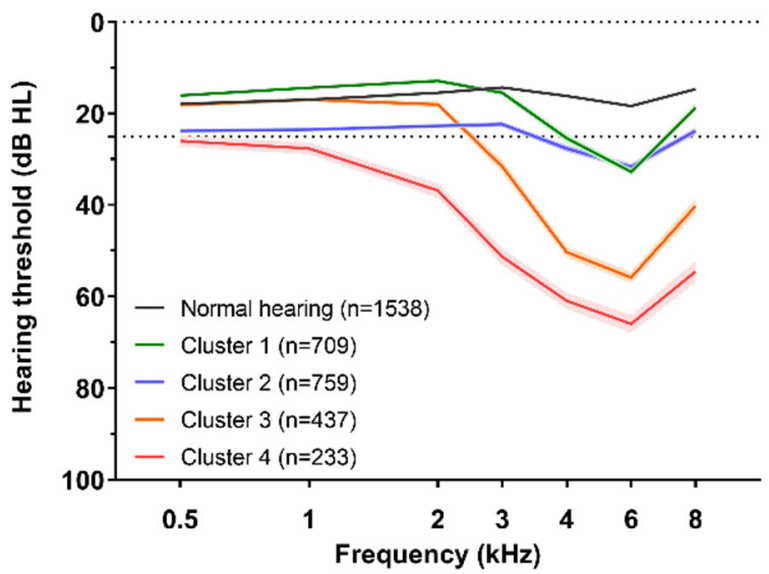

FIGURE 2 | Optimal clusters for three datasets. The within cluster sum of squares (WSS) decrease with the increment of clusters number, and the optimal number of clustering was selected at the last one significantly reduced the WSS (at the inflection point of the curve). The optimal clustering were all at number of four (black dotted line) for total dataset (A), dataset 1 (C), and dataset 2 (E). The average hearing thresholds over $0.5-8 \mathrm{kHz}$ frequencies of normal hearing subjects and those four clusters were shown for total dataset (B), dataset 1 (D), and dataset 2 (F). 
TABLE 1 | Characteristics of subjects in different datasets.

\begin{tabular}{|c|c|c|c|c|}
\hline Variables & Total $(n=10,307)$ & Dataset $1(n=6,631)$ & Dataset $2(n=3,676)$ & $P$ value ${ }^{*}$ \\
\hline Age (years), mean (SD) & $34.5(8.8)$ & $36.2(8.6)$ & $31.4(8.3)$ & $<0.001$ \\
\hline CNE (dB[A]), mean (SD) & $91.2(22.7)$ & $92.0(22.0)$ & $89.8(23.9)$ & $<0.001$ \\
\hline Males & $9,165(88.9)$ & $5,855(88.3)$ & $3,310(90.0)$ & \\
\hline Females & $1,142(11.1)$ & $776(11.7)$ & $366(10.0)$ & \\
\hline Obese & $936(9.1)$ & $631(9.5)$ & $305(8.3)$ & \\
\hline Hearing difficulty, $n(\%)$ & & & & $<0.001$ \\
\hline No & $7,955(77.2)$ & $4,952(74.7)$ & 3,003 (81.7) & \\
\hline Yes & 2,352 (22.8) & $1,679(25.3)$ & 673 (18.3) & \\
\hline Tinnitus, $n(\%)$ & & & & $<0.001$ \\
\hline$<4$ h/work-day & $7,384(71.6)$ & $4,936(74.4)$ & $2,448(66.6)$ & \\
\hline$\geq 4$ h/work-day & $2,923(28.4)$ & $1,695(25.6)$ & $1,228(33.4)$ & \\
\hline Earphone use, $n(\%)$ & & & & $<0.001$ \\
\hline$<1 \mathrm{~h} /$ day & $5,844(56.7)$ & $3,418(51.5)$ & $2,426(66.0)$ & \\
\hline$\geq 1 \mathrm{~h} /$ day & 4,463 (43.3) & $3,213(48.5)$ & $1,250(34.0)$ & \\
\hline Tobacco consumption, $n$ (\%) & & & & 0.156 \\
\hline$<10$ cigarettes/day & $6,436(62.4)$ & $4,174(62.9)$ & 2,262 (61.5) & \\
\hline$\geq 10$ cigarettes/day & $3,871(37.6)$ & $2,457(37.1)$ & $1,414(38.5)$ & \\
\hline Alcohol consumption, $n$ (\%) & & & & $<0.001$ \\
\hline$<50$ g/day & 7,558 (73.3) & $5,076(76.5)$ & 2,482 (67.5) & \\
\hline$\geq 50$ g/day & $2,749(26.7)$ & $1,555(23.5)$ & $1,194(32.5)$ & \\
\hline
\end{tabular}

$S D$, standard deviation; BMI, body mass index; CNE, cumulative noise exposure; HPD, hearing protective device.

${ }^{*}$ Comparisons were between dataset 1 and dataset 2.

all models, odds ratios (ORs) and 95\% confidence intervals (CIs) are presented. A 2-tailed $P<0.05$ was considered statistically significant.

\section{RESULTS}

\section{Basic Characteristics of Subjects}

A total of 10,307 Chinese Han subjects (9,165 males [88.9\%], mean age 34.5 [SD 8.8] years, mean CNE 91.2 [SD 22.7] $\mathrm{dB}[\mathrm{A}])$ were included. Among all subjects, 3,708 (36.0\%) had completely normal hearing over $0.5-8 \mathrm{kHz}$ frequencies. The total subjects were recruited from two independent factories in a shipyard, who had similar types of occupational tasks, despite significantly different distributions of sex, age, CNE, hearing loss, and other characteristics. The distributions of age, CNE, sex, BMI, hearing difficulty, tinnitus, HPD use, earphone use, tobacco consumption, and alcohol consumption are shown in Table 1.

\section{Clusters of Audiometric Phenotypes}

To classify NIHL into novel audiometric phenotypes, we used the k-means clustering method in audiograms with hearing loss. We repeated the cluster process, respectively, in total dataset (all the hearing loss audiograms, $n=6,599$ ), dataset 1 (hearing loss audiograms from factory $1, n=4,461$ ) and dataset 2 (hearing loss audiograms from factory $2, n=2,138$ ) to verify that the cluster structure described for each dataset was reproducible.

For all three datasets, the optimal number of clusters was four according to the WSS decreasing curve (Figures 2A-E), and the audiometric phenotypes of four clusters identified from different datasets were qualitatively similar. In total, 6,239 /6,599 (94.5\%) audiograms in total dataset clusters were classified into the same subtype according to the distinct clusters in dataset $1(4,286 / 4,461,96.1 \%)$ and dataset $2(1,953 / 2,138,91.3 \%)$, the consistency of subtypes by cluster analysis in two distinct datasets and total dataset showed in Table 2. The average hearing thresholds of normal hearing subjects and those four clusters are shown for each dataset (Figures 2B-F). 
TABLE 2 | The consistency of subtypes by cluster analysis in two independent datasets and total dataset.

\begin{tabular}{|c|c|c|c|c|c|}
\hline Consistency, $n(\%)$ & Cluster 1 & Cluster 2 & Cluster 3 & Cluster 4 & Total \\
\hline Dataset 1 & $1,549(100.0)$ & 1,123 (89.9) & 1,075 (97.3) & 539 (96.6) & 4,286 (96.1) \\
\hline Dataset 2 & 703 (91.4) & $737(94.0)$ & 354 (83.1) & 159 (100.0) & 1,953 (91.3) \\
\hline Total & 2,252 (97.2) & 1,860 (91.5) & 1,492 (93.3) & 698 (97.4) & $6,239(94.5)$ \\
\hline
\end{tabular}

\section{Relevant Characteristics of Audiometric Phenotypes}

Audiograms with hearing loss were then classified into 4 subtypes for cluster analysis of the total dataset, which were named $4-6 \mathrm{kHz}$ sharp-notched (original cluster 1, Figure 3A), $4-6 \mathrm{kHz}$ flat-notched (original cluster 2, Figure 4A), $3-8 \mathrm{kHz}$ notched (original cluster 3 , Figure 5A), and $1-8 \mathrm{kHz}$ notched (original cluster 4, Figure 6A) phenotypes, referring to the frequency range, and shape of their audiometric notches. Hearing thresholds at frequencies of $0.5-8 \mathrm{kHz}$ of the four subtypes were significantly different from each other (all the $P$ values $<0.001)$. In comparison with the $4-6 \mathrm{kHz}$ sharp- and flatnotched subtypes, subjects manifested as the $3-8 \mathrm{kHz}$ and $1-$ $8 \mathrm{kHz}$ notched subtypes were significantly older, with higher noise exposure, as well as higher proportions of males, hearing difficulties and tinnitus. In post-hoc analyses, for the $4-6 \mathrm{kHz}$ flatnotched audiogram, the average age of subjects of this subtype was similar $(P=0.293)$ to that of the $4-6 \mathrm{kHz}$ sharp-notched audiogram, while the mean $\mathrm{CNE}$ was slightly smaller $(P=$ 0.008 ) than that of the $4-6 \mathrm{kHz}$ sharp-notched audiogram, but significantly larger $(P<0.001)$ than that of the normal-hearing audiogram. The proportions of females, hearing difficulties, tinnitus, and earphone uses were higher in subjects with the 4$6 \mathrm{kHz}$ flat-notched audiogram than that in the $4-6 \mathrm{kHz}$ sharpnotched audiogram. Moreover, the average hearing thresholds of the $4-6 \mathrm{kHz}$ flat-notched audiogram at frequencies of $0.5-3 \mathrm{kHz}$ were obviously higher than that of the $4-6 \mathrm{kHz}$ sharp-notched audiogram (all the $P$ value $<0.001$ ). The detailed distribution of characteristics in subjects with different audiometric phenotypes is shown in Table 3.

Variables that showed significant differences between audiometric phenotypes were included in the multinomial logistic regression analysis (Table 4). Age, male sex, tobacco consumption, and alcohol consumption were risk factors for all subtypes, while the HPD use was a protective factor. CNE was associated with three of all subtypes except for the $4-6 \mathrm{kHz}$ flat-notched phenotype. Tinnitus was associated with three of all subtypes except for the $4-6 \mathrm{kHz}$ sharp-notched phenotype. Self-reported hearing difficulty was only related to the $1-8 \mathrm{kHz}$ notched phenotype, which reflected the most severe NIHL subtype.

\section{Specific Influence of Noise Exposure on Audiometric Phenotypes}

To explore the specific influence of noise exposure dose on audiometric phenotypes among populations with different characteristics, we performed hierarchical regression analysis

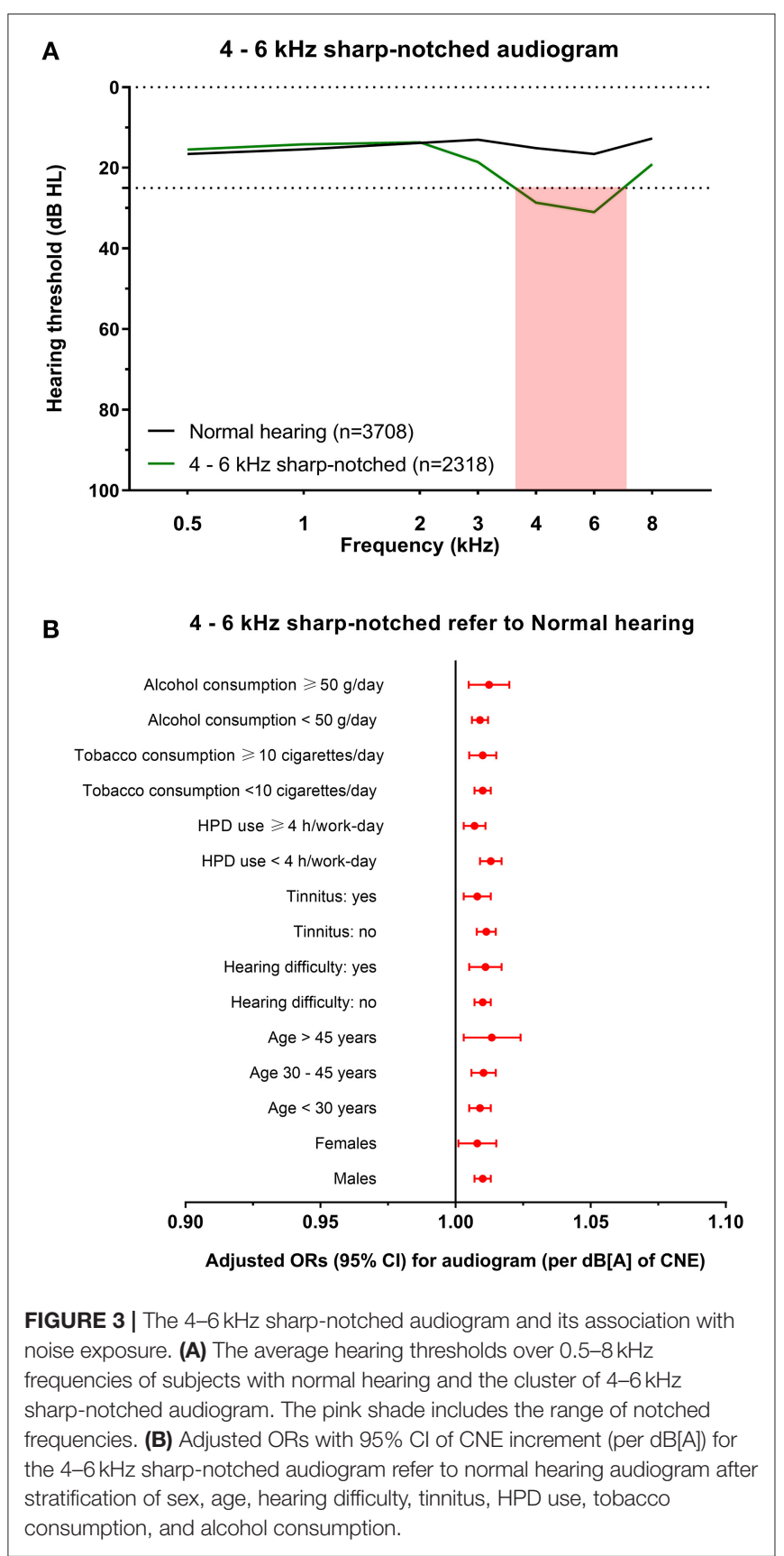

of audiometric phenotypes stratified by confounding factors (sex, age, CNE, HPD use, hearing difficulty, tinnitus, tobacco consumption, and alcohol consumption). According to 


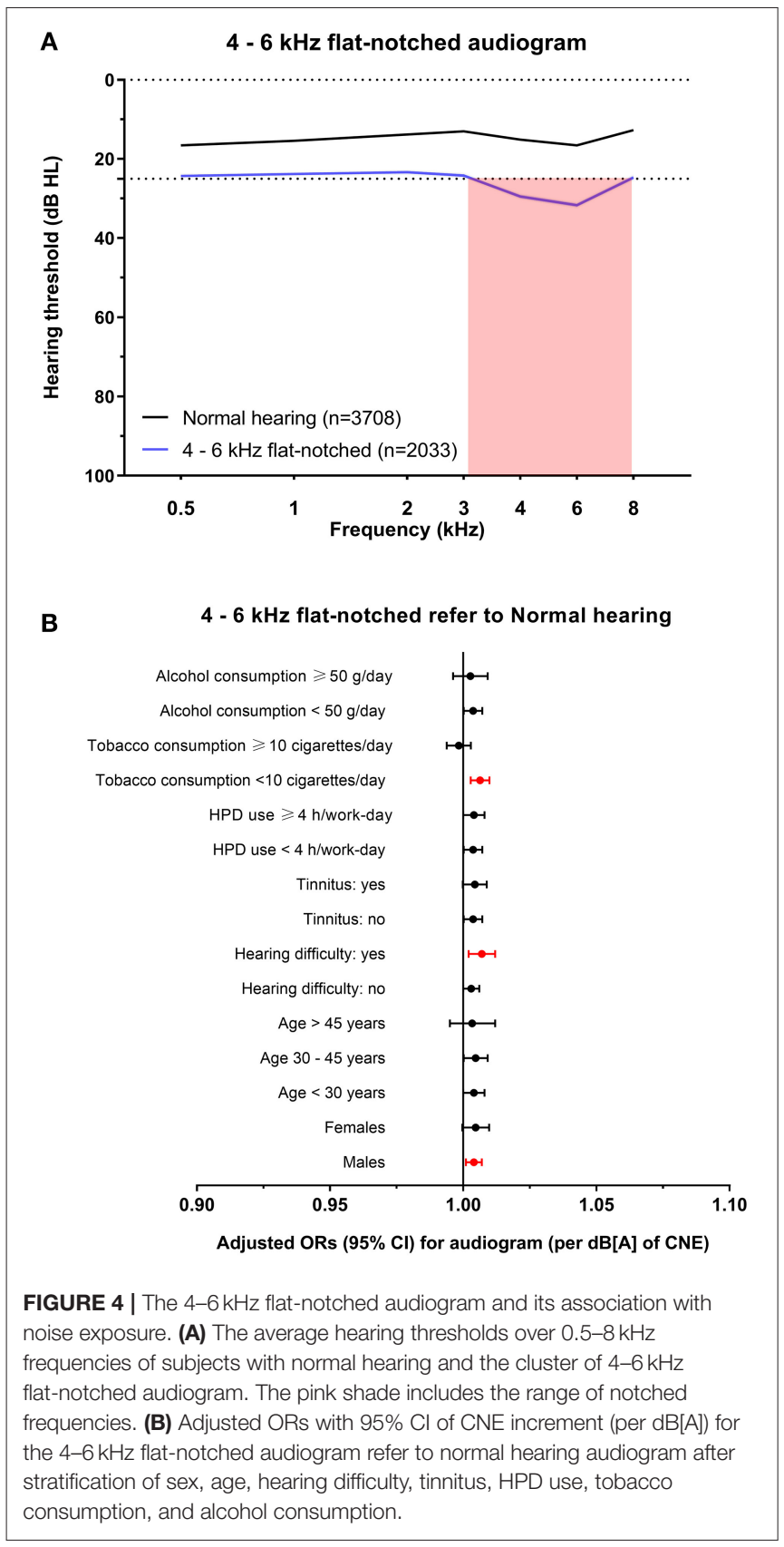

the adjusted ORs of noise exposure dose for different phenotypes after stratification, the increment of CNE was stably associated with the $4-6 \mathrm{kHz}$ sharp-notched phenotype (Figure 3B), as well as associated with the 3$8 \mathrm{kHz}$ notched phenotype except among younger subjects $(<30$ years old) (Figure 5B) and the $1-8 \mathrm{kHz}$ notched phenotypes except for females and younger population (Figure 6B). In contrast, $\mathrm{CNE}$ was almost unrelated to the $4-6 \mathrm{kHz}$ flat-notched phenotype (Figure 4B), except for population who were males, with hearing difficulty and little tobacco consumption.

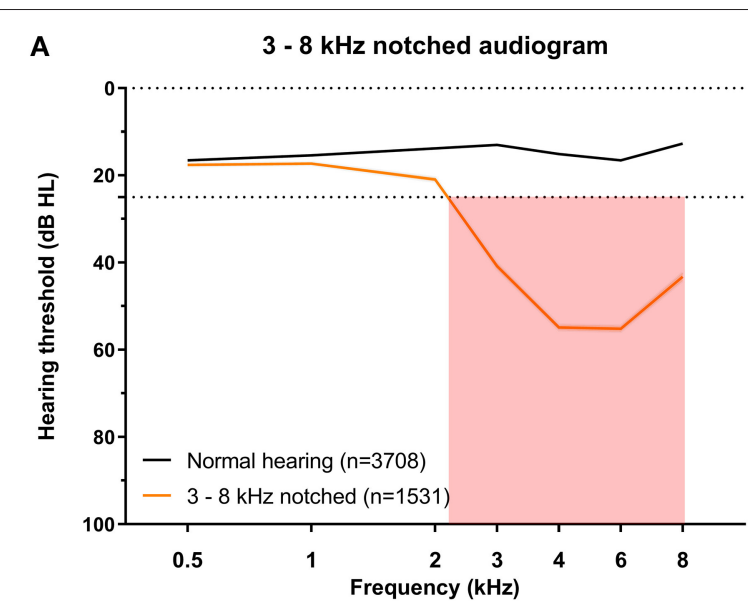

B

\section{3 - $8 \mathrm{kHz}$ notched refer to Normal hearing}

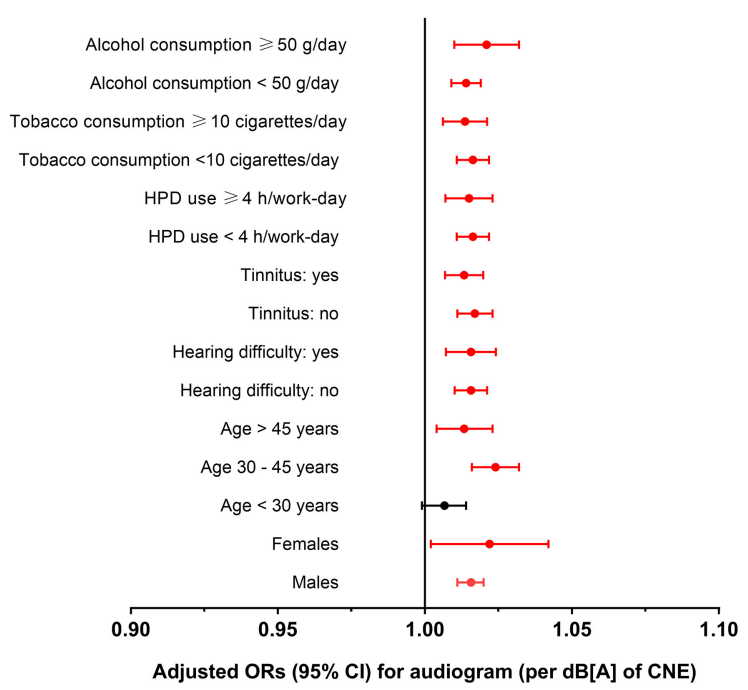

FIGURE 5 | The 3-8 kHz notched audiogram and its association with noise exposure. (A) The average hearing thresholds over $0.5-8 \mathrm{kHz}$ frequencies of subjects with normal hearing and the cluster of $3-8 \mathrm{kHz}$ notched audiogram. The pink shade includes the range of notched frequencies. (B) Adjusted ORs with $95 \% \mathrm{Cl}$ of $\mathrm{CNE}$ increment (per dB[A]) for the $3-8 \mathrm{kHz}$ notched audiogram refer to normal hearing audiogram after stratification of sex, age, hearing difficulty, tinnitus, HPD use, tobacco consumption, and alcohol consumption.

\section{DISCUSSION}

In this study we performed a cluster analysis of noise-exposed population who had some degree of hearing loss. By using the audiometric thresholds over $0.5-8 \mathrm{kHz}$ of the total hearing loss dataset $(n=6,599)$, we developed the cluster model and identified four phenotypes with distinct audiogram subtypes of hearing loss. We repeated the cluster analysis in two independent parts of the total dataset, dataset $1(n=4,461)$ and dataset $2(n=2,138)$ where we were able to replicate the clusters into four similar phenotypes. The relevant demographic and behavioral characteristics of population with different hearing 


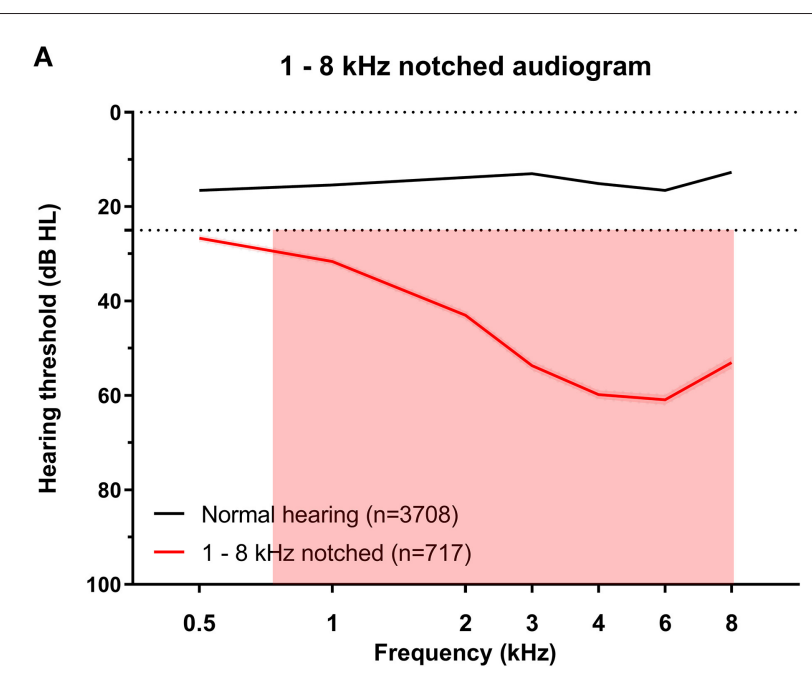

B
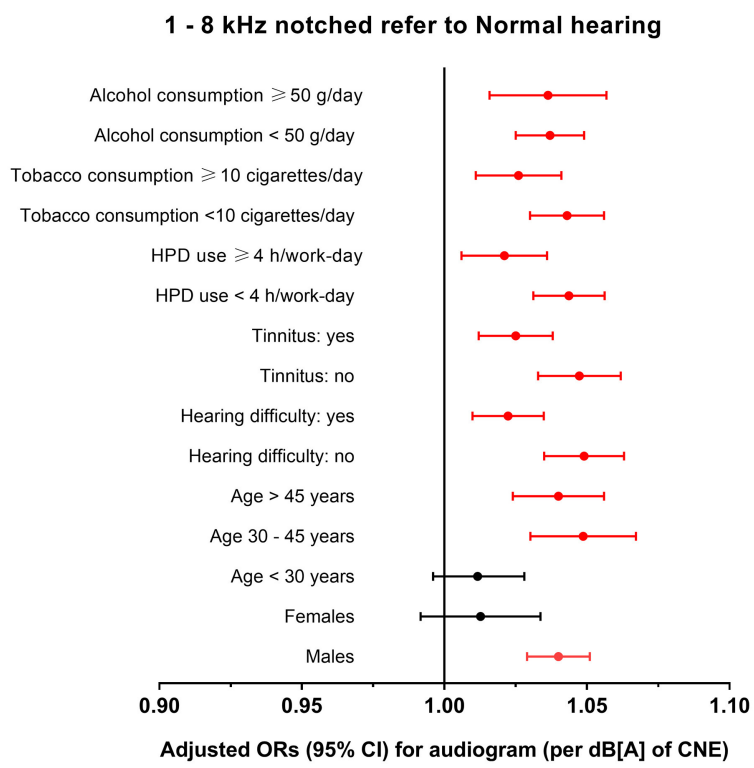

FIGURE 6 | The $1-8 \mathrm{kHz}$ notched audiogram and its association with noise exposure. (A) The average hearing thresholds over $0.5-8 \mathrm{kHz}$ frequencies of subjects with normal hearing and the cluster of $1-8 \mathrm{kHz}$ notched audiogram. The pink shade includes the range of notched frequencies. (B) Adjusted ORs with $95 \% \mathrm{Cl}$ of $\mathrm{CNE}$ increment (per dB[A]) for the $1-8 \mathrm{kHz}$ notched audiogram refer to normal hearing audiogram after stratification of sex, age, hearing difficulty, tinnitus, HPD use, tobacco consumption, and alcohol consumption.

loss phenotypes were analyzed in comparison with the normal hearing population ( $n=3,708)$.

Our main finding was that hearing loss in noise-exposed population consisted of four audiogram subtypes that had different characteristics and associations with noise exposure levels. In line with previous studies, we found the presence of a "notch" at high frequencies of 3,4 , and $6 \mathrm{kHz}$ with recovery at $8 \mathrm{kHz}$ in most hearing loss audiograms, some of which extended to involve even $1 \mathrm{kHz}$ and $2 \mathrm{kHz}(14-16)$. Therefore, we named the phenotypes $4-6 \mathrm{kHz}$ sharp-notched, $4-6 \mathrm{kHz}$ flat-notched, $3-8 \mathrm{kHz}$ notched, and $1-8 \mathrm{kHz}$ notched audiograms.

In the present study, the $4-6 \mathrm{kHz}$ sharp-notched audiogram, $3-8 \mathrm{kHz}$ notched audiogram, and $1-8 \mathrm{kHz}$ notched audiogram were strongly related to noise exposure, which represented three distinct subtypes of NIHL. This result supported the conventional description of noise-induced high-frequency audiometric notches $(8,9,23)$ based on data-driven evidence. The occurrence of $4-6 \mathrm{kHz}$ sharp-notched audiogram was highest among all subtypes with constant correlation to the noise exposure, which could be regarded as a typical subtype of NIHL. While the $3-8 \mathrm{kHz}$ notched audiogram and $1-8 \mathrm{kHz}$ notched audiogram that manifested as more severe subtypes of NIHL involved wider ranges of frequency, which were less likely to appear among younger populations and even females. This finding agreed with several previous studies suggesting that the risk of NIHL in males was significantly higher than that in females $(12,15,24)$, as well as the effects of aging may extend the hearing loss frequencies to $8 \mathrm{kHz}$ and even low frequencies, which reduces the prominence of the typical "notch" in audiograms of individuals with excess noise exposure $(8,9)$.

In particular, the $4-6 \mathrm{kHz}$ flat-notched audiogram was the second most common subtype of hearing loss after the 4$6 \mathrm{kHz}$ sharp-notched audiogram, however, it seemed to be unrelated to noise exposure, but associated with age, sex, and some behavioral factors according to the logistic regression (Table 4). Although the mean CNE of subjects with the 4$6 \mathrm{kHz}$ flat-notched audiogram was significantly larger than that of the normal-hearing audiogram, it might due to the longer working-length of subjects with the $4-6 \mathrm{kHz}$ flatnotched audiogram, who were also older than those with the normal-hearing audiogram. In addition, the average hearing thresholds of the $4-6 \mathrm{kHz}$ flat-notched audiogram at lower frequencies were higher than that of the $4-6 \mathrm{kHz}$ sharp-notched audiogram, despite of the similar mean age, and CNE. However, in consideration of the obvious differences in sex, hearing difficulty, tinnitus, and earphone use between the two subtypes, we speculated that there should be other factors (such as individual behaviors and genetic heterogeneity) influencing the audiometric phenotypes, which should be further explored in future studies. This finding may provide an explanation for some previous studies reporting that audiometric notches also commonly occur in individuals without any previous noise exposure and have been associated with other factors (14, 15, 25). The Nord-Trøndelag Hearing Loss Study analyzed the various definitions of notched audiograms in the 3$6 \mathrm{kHz}$ range [defined by Coles et al. (9), Hoffman et al. (26), Wilson and Mcardle (27)] in 49774 subjects aged 20-101 years. The prevalence of those notches varied from 60 to $70 \%$ in the most noise-exposed men, but was also common in men without any occupational noise exposure. Another study using the Hoffmann notch to analyze audiograms of US adults from the NHANES (16) showed that though 8.2\% of 1,223 self-reported occupational noise-exposed individuals had bilateral high-frequency audiometric notches, $5.2 \%$ of 2,360 individuals without noise exposure also had bilateral notches. Those artificial definitions of notches probably included 
TABLE 3 | Characteristics of subjects in different audiometric phenotypes.

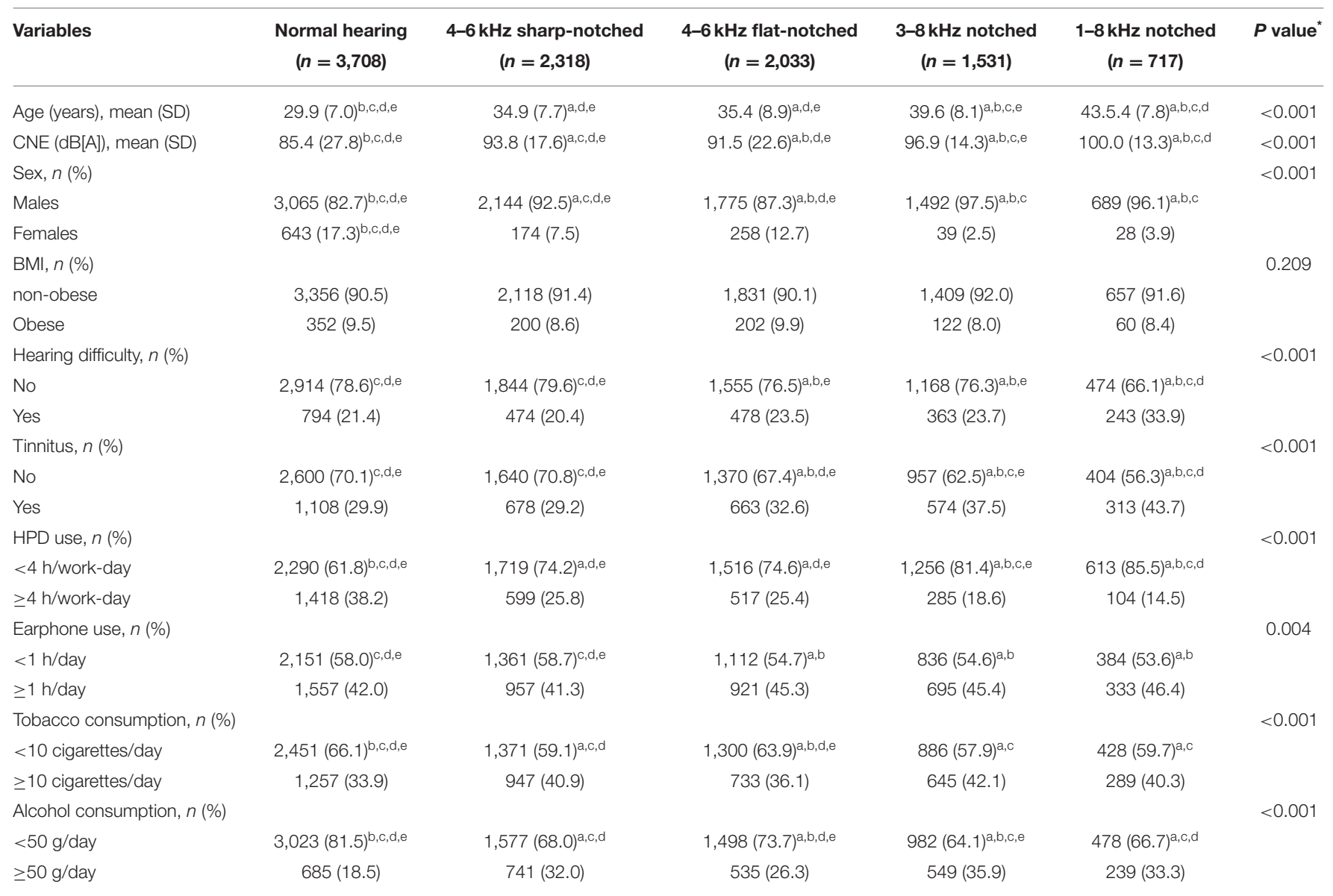

$S D$, standard deviation; BMI, body mass index; CNE, cumulative noise exposure; HPD, hearing protective device.

${ }^{*}$ Comparisons were between different audiometric phenotypes.

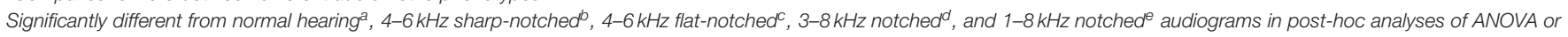
chi-square test $(P<0.05)$.

TABLE 4 | Multinomial logistic regression models of audiometric phenotypes.

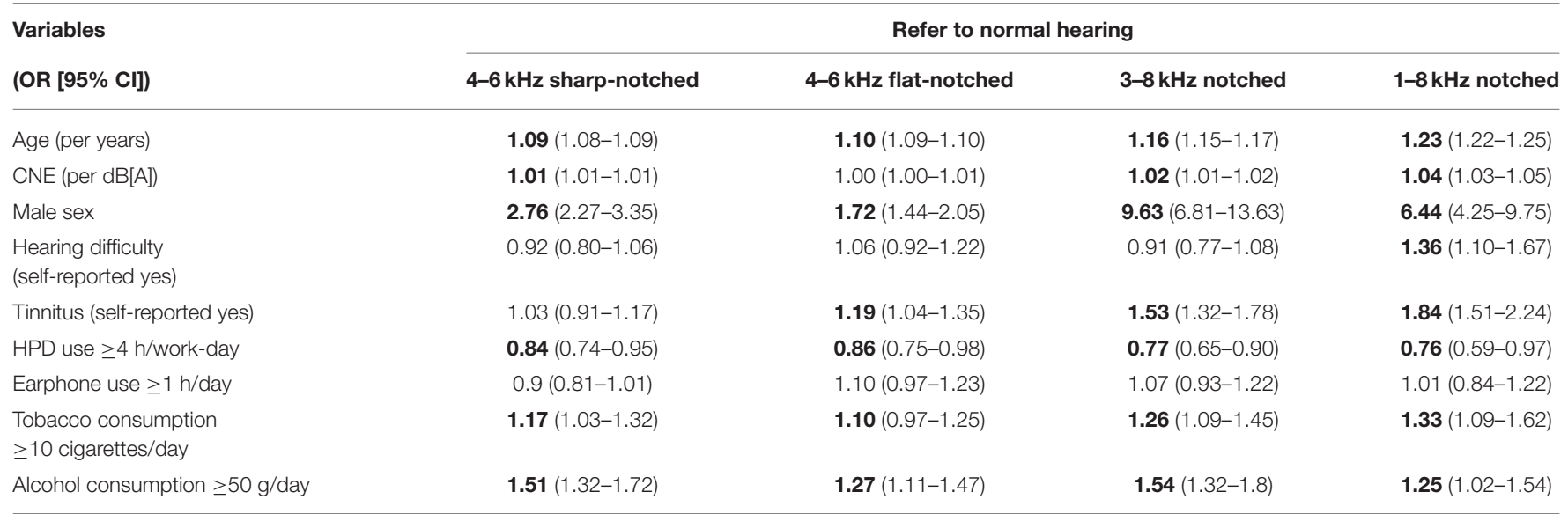

Reference variables: Sex, females; Hearing difficulty, self-reported no; Tinnitus, self-reported no; HPD use, $<4$ h/work day; Earphone use, $<1$ h/day; Tobacco consumption, $<10$ cigarettes/day; Alcohol consumption, $<50 \mathrm{~g} /$ day.

Bold type: $P<0.05$.

$\mathrm{OR}$, odds ratio; $\mathrm{Cl}$, confidence interval; $B M I$, body mass index; $C N E$, cumulative noise exposure; $H P D$, hearing protective device. 
this $4-6 \mathrm{kHz}$ flat-notched audiogram, which may limit the specificity of using high-frequency audiometric notch for the diagnosis of NIHL.

As many previous studies reported $(11,28,29)$, we found that age, sex, tobacco, and alcohol consumption were confounding influencing factors of hearing loss other than noise exposure. Using HPDs in an environment with loud noise exposure for hours every work-day likely protected individuals from NIHL, despite audiometric subtypes. In addition, we found that tinnitus was associated with the degree of hearing loss rather than the most typical NIHL subtype, while self-reported hearing difficulty was only closely related to the most severe subtype of hearing loss with speech frequencies impairment. These findings are approximately consistent with previous studies that reported that tinnitus is usually accompanied by hearing loss (30), and self-reported hearing status could not sensitively reflect high-frequency hearing loss (31).

It is widely accepted that audiometric phenotypes are based on presumed underlying auditory histopathology, which suggests the causes and degree of auditory organ damage (32, 33). A few previous studies have performed cluster analysis in clinical audiograms. Interestingly, the notched audiometric phenotype was always distinguished out as a separate cluster $(18,19)$, and we assumed that it should indicate the NIHL phenotype, although the noise exposure history of those patients was not reported. Here we propose to use this cluster classification to identify audiometric phenotypes for the evaluation of NIHL, since the typical NIHL in a specific population may manifest as different subtypes of notched audiograms, and suggest different management approaches. For instance, the presence of $4-6 \mathrm{kHz}$ sharp-notched audiogram in younger females might be a strong signal indicating NIHL, in contrast, the $4-6 \mathrm{kHz}$ flat-notched audiogram should not be evidence of NIHL. This would facilitate optimal assessment of NIHL.

The main strength of our study is that it first provides various reproducible audiometric subtypes of NIHL by data-driven analysis in a relatively large-scale noise-exposed population. Another strength was that our study was based on consideration of detailed noise exposure history, questionnaire information and audiometric data from standardized protocols, which can give a more nuanced picture than clinical data. Previously Zhao et al. developed machine learning models for the prediction of NIHL (34), which were based on hypothesis-driven or supervised analysis. Instead, for the first time to our knowledge, we performed an unsupervised data-driven cluster analysis to identify the unknown audiometric phenotypes associated with noise exposure, and to describe the relevant characteristics of distinct subtypes of NIHL. However, there are also some limitations. First, this cross-sectional study did not allow robust causal inference, although the employees were supposed to have a pre-work health examination to ensure normal hearing at baseline. Second, all subjects in this study were collected in the same region of China and they may not represent the whole noise-exposed population. Furthermore, we cannot at this stage claim that the new subtypes represent different etiologies of NIHL, or that this clustering is the optimal classification of NIHL phenotypes.

In conclusion, we were able to repeat and identify distinct audiometric phenotypes of NIHL in large-scale noiseexposed populations with different relevant characteristics, by using cluster analysis. Moreover, given the technological advances in machine learning, our study provides a sight into the prospect of involving data-driven audiogram mining for the precise diagnosis and treatment of NIHL in future studies.

\section{DATA AVAILABILITY STATEMENT}

The original contributions presented in the study are included in the article/supplementary material, further inquiries can be directed to the corresponding authors.

\section{ETHICS STATEMENT}

The studies involving human participants were reviewed and approved by the Ethics Committee of the Ninth People's Hospital affiliated to Shanghai Jiao Tong University School of Medicine. The patients/participants provided their written informed consent to participate in this study.

\section{AUTHOR CONTRIBUTIONS}

QW, MQ, ZH, and HW: contributed to the study design. QW and MQ: writing the original draft. MQ, LY, JS, YH, and $\mathrm{KH}$ : contributed to the acquisition of data. QW, CL, and JL contributed to the analysis of data, $\mathrm{ZH}$ and HW contributed to the supervision and funding acquisition of the work, review and revision of the draft. All authors contributed to the interpretation of data and critical revision of the draft. All authors gave final approval of the version to be published.

\section{FUNDING}

This study was supported by grant 81730028 to HW from the National Natural Science Foundation of China, grant 2017YFC1001804 to HW from the National Key Research and Development Program of China. This work was also supported by grant 20ZR1431200 to $\mathrm{ZH}$ from the Natural Science Foundation of Shanghai, grant YG2021ZD14 to ZH from the Medical Engineering Cross Research Funding of Shanghai Jiao Tong University.

\section{ACKNOWLEDGMENTS}

We thank all the subjects for their participation and the security department of shipyard for the cooperation. 


\section{REFERENCES}

1. Masterson EA, Bushnell PT, Themann CL, Morata TC. Hearing impairment among noise-exposed workers - United States, 2003-2012. MMWR Morb Mortal Wkly Rep. (2016) 65:389-94. doi: 10.15585/mmwr.mm6515a2

2. Eichwald J, Scinicariello F. Survey of teen noise exposure and efforts to protect hearing at school United States, 2020. MMWR Morb Mortal Wkly Rep. (2020) 69:1822-6. doi: 10.15585/mmwr.mm6948a5

3. Yao C, Ma AK, Cushing SL, Lin VYW. Noise exposure while commuting in Toronto - a study of personal and public transportation in Toronto. $J$ Otolaryngol Head Neck Surg. (2017) 46:62. doi: 10.1186/s40463-017-0239-6

4. Eichwald J, Scinicariello F, Telfer JL, Carroll YI. Use of personal hearing protection devices at loud athletic or entertainment events among adults - United States, 2018. MMWR Morb Mortal Wkly Rep. (2018) 67:1151-5. doi: $10.15585 / \mathrm{mmwr} . \mathrm{mm} 6741 \mathrm{a} 4$

5. Basner M, Babisch W, Davis A, Brink M, Clark C, Janssen S, et al. Auditory and non-auditory effects of noise on health. Lancet. (2014) 383:1325-32. doi: 10.1016/S0140-6736(13)61613-X

6. Wilson BS, Tucci DL, Merson MH, O'donoghue GM. Global hearing health care: new findings and perspectives. Lancet. (2017) 390:2503-15. doi: 10.1016/S0140-6736(17)31073-5

7. Lutman ME, Coles RR, Buffin JT. Guidelines for quantification of noiseinduced hearing loss in a medicolegal context. Clin Otolaryngol. (2016) 41:347-57. doi: 10.1111/coa.12569

8. Mirza R, Kirchner DB, Dobie RA, Crawford J. Occupational noiseinduced hearing loss. J Occup Environ Med. (2018) 60:e498-e501. doi: $10.1097 / J O M .0000000000001423$

9. Coles RR, Lutman ME, Buffin JT. Guidelines on the diagnosis of noiseinduced hearing loss for medicolegal purposes. Clin Otolaryngol Allied Sci. (2000) 25:264-73. doi: 10.1046/j.1365-2273.2000.00368.x

10. Ali S, Morgan M, Ali UI. Is it reasonable to use 1 and $8 \mathrm{kHz}$ anchor points in the medico-legal diagnosis and estimation of noise-induced hearing loss? Clin Otolaryngol. (2015) 40:255-9. doi: 10.1111/coa.12362

11. Morris RJ. On the need for an "Industry-Wide Standard" definition of an audiometric notch and the lack of specificity of the audiometric notch in the diagnosis of noise-induced hearing loss. J Occup Environ Med. (2019) 61:e226. doi: 10.1097/JOM.0000000000001550

12. Lie A, Skogstad M, Johnsen TS, Engdahl B, Tambs K. A cross-sectional study of hearing thresholds among 4627 Norwegian train and track maintenance workers. BMJ Open. (2014) 4:e005529. doi: 10.1136/bmjopen-2014-005529

13. Peng Y, Fan C, Hu L, Peng S, Xie P, Wu F, et al. Tunnel driving occupational environment and hearing loss in train drivers in China. Occup Environ Med. (2019) 76:97-104. doi: 10.1136/oemed-2018-105269

14. Nondahl DM, Shi X, Cruickshanks KJ, Dalton DS, Tweed TS, Wiley TL, et al. Notched audiograms and noise exposure history in older adults. Ear Hear. (2009) 30:696-703. doi: 10.1097/AUD.0b013e3181b1d418

15. Lie A, Skogstad M, Johnsen TS, Engdahl B, Tambs K. The prevalence of notched audiograms in a cross-sectional study of 12,055 railway workers. Ear Hear. (2015) 36:e86-92. doi: 10.1097/AUD.0000000000000129

16. Carroll YI, Eichwald J, Scinicariello F, Hoffman HJ, Deitchman S, Radke MS, et al. Vital signs: noise-induced hearing loss among adults United States 2011-2012. MMWR Morb Mortal Wkly Rep. (2017) 66:139-44. doi: $10.15585 / \mathrm{mmwr}$ mm6605e3

17. Lisboa PJ, Etchells TA, Jarman IH, Chambers SJ. Finding reproducible cluster partitions for the k-means algorithm. BMC Bioinformatics. (2013) 14(Suppl 1):S8. doi: 10.1186/1471-2105-14-S1-S8

18. Lee CY, Hwang JH, Hou SJ, Liu TC. Using cluster analysis to classify audiogram shapes. Int $J$ Audiol. (2010) 49:628-33. doi: 10.3109/14992021003796887

19. Parthasarathy A, Romero Pinto S, Lewis RM, Goedicke W, Polley DB. Data-driven segmentation of audiometric phenotypes across a large clinical cohort. Sci Rep. (2020) 10:6704. doi: 10.1038/s41598-02063515-5

20. Zhao YM, Qiu W, Zeng L, Chen SS, Cheng XR, Davis RI, et al. Application of the kurtosis statistic to the evaluation of the risk of hearing loss in workers exposed to high-level complex noise. Ear Hear. (2010) 31:527-32. doi: 10.1097/AUD.0b013e3181d94e68

21. R Foundation for Statistical Computing. $R: A$ Language and Environment for Statistical Computing. Austria (2020). Available online at: https://www.Rproject.org/ (accessed December 20, 2020).

22. Steinley D, Brusco MJ. Choosing the number of clusters in $\kappa$-means clustering. Psychol Methods. (2011) 16:285-97. doi: 10.1037/a0023346

23. Mcbride DI, Williams S. Audiometric notch as a sign of noise induced hearing loss. Occup Environ Med. (2001) 58:46-51. doi: 10.1136/oem.58.1.46

24. Bhatt IS, Guthrie O. Analysis of audiometric notch as a noise-induced hearing loss phenotype in US youth: data from the National Health And Nutrition Examination Survey, 2005-2010. Int J Audiol. (2017) 56:392-9. doi: 10.1080/14992027.2017.1278799

25. Osei-Lah V, Yeoh LH. High frequency audiometric notch: an outpatient clinic survey. Int J Audiol. (2010) 49:95-8. doi: 10.3109/14992020903300423

26. Hoffman HJ, Ko CW, Themann CL, Dillon CF, Franks JR. Reducing Noiseinduced Hearing Loss (NIHL) to achieve us healthy people 2010 goals. Am J Epidemiol. (2006) 163:S122. doi: 10.1093/aje/163.suppl_11.S122-c

27. Wilson RH, Mcardle R. Characteristics of the audiometric $4,000 \mathrm{~Hz}$ notch $(744,553$ veterans) and the $3,000,4,000$, and $6,000 \mathrm{~Hz}$ notches. (539,932 veterans). J Rehabil Res Dev. (2013) 50:111-32. doi: 10.1682/JRRD.2011.11.0225

28. Tikka C, Verbeek JH, Kateman E, Morata TC, Dreschler WA, Ferrite S. Interventions to prevent occupational noise-induced hearing loss. Cochrane Database Syst Rev. (2017) 7:Cd006396. doi: 10.1002/14651858.CD006396.pub4

29. Golmohammadi R, Darvishi E. The combined effects of occupational exposure to noise and other risk factors - a systematic review. Noise Health. (2019) 21:125-41. doi: 10.4103/nah.NAH_4_18

30. Baguley D, Mcferran D, Hall D. Tinnitus. Lancet. (2013) 382:1600-7. doi: 10.1016/S0140-6736(13)60142-7

31. Tsimpida D, Kontopantelis E, Ashcroft D, Panagioti M. Comparison of selfreported measures of hearing with an objective audiometric measure in adults in the english longitudinal study of ageing. JAMA Netw Open. (2020) 3:e2015009. doi: 10.1001/jamanetworkopen.2020.15009

32. Schmiedt RA, Lang H, Okamura HO, Schulte BA. Effects of furosemide applied chronically to the round window: a model of metabolic presbyacusis. J Neurosci. (2002) 22:9643-50. doi: 10.1523/JNEUROSCI.22-21-09643.2002

33. Demeester K, Van Wieringen A, Hendrickx JJ, Topsakal V, Fransen E, Van Laer L, et al. Audiometric shape and presbycusis. Int J Audiol. (2009) 48:22232. doi: 10.1080/14992020802441799

34. Zhao Y, Li J, Zhang M, Lu Y, Xie H, Tian Y, et al. Machine learning models for the hearing impairment prediction in workers exposed to complex industrial noise: a pilot study. Ear Hear. (2019) 40:690-9. doi: 10.1097/AUD.0000000000000649

Conflict of Interest: The authors declare that the research was conducted in the absence of any commercial or financial relationships that could be construed as a potential conflict of interest.

Copyright (๑ 2021 Wang, Qian, Yang, Shi, Hong, Han, Li, Lin, Huang and Wu. This is an open-access article distributed under the terms of the Creative Commons Attribution License (CC BY). The use, distribution or reproduction in other forums is permitted, provided the original author(s) and the copyright owner(s) are credited and that the original publication in this journal is cited, in accordance with accepted academic practice. No use, distribution or reproduction is permitted which does not comply with these terms. 